\title{
Bi Polar Q-Fuzzy Left R -Subgroups of Near-Rings
}

\author{
S.Subramanian \\ Assistant Professor \\ Department of Mathematics \\ Saranathan College of Engineering \\ Tiruchirappalli- 12. \\ Tamil Nadu, India.
}

\author{
R.Nagarajan \\ Assistant Professor \\ Department of Mathematics \\ J.J.College of Engineering \\ Trichirappally-09. \\ Tamil Nadu India.
}

\author{
B.Chellappa \\ Reader in Mathematics \\ Department of Mathematics \\ Alagappa Govt Arts College \\ Karaikudi. \\ Tamil Nadu, India.
}

\begin{abstract}
In this paper, we introduce the notion of Q- fuzzification of Bipolar left R- subgroups in a near-ring and investigate some related properties. Characterization of Bipolar Q- fuzzy left Rsubgroups with respect to(T,S)are given.
\end{abstract}

Mathematics subject classification: 03F055, $03 E 72$.

Index terms: Q- fuzzy set, Q- fuzzy R- subgroup (sub near rings), Bipolar Fuzzy set, Bipolar Q- fuzzy left R- subgroups, $(\mathrm{T}, \mathrm{S})$ norm.

\section{INTRODUCTION}

Fuzzy sets are a kind of useful mathematical structure to represent a collection of objects whose boundary is vague. There are several kinds of fuzzy sets extensions in the fuzzy set theory, for example, intuitionistic fuzzy sets, interval valued fuzzy sets, vague sets etc. Bipolar-valued fuzzy sets are an extension of fuzzy sets whose membership degree range is enlarged from the interval $[0,1]$ to $[-1,1]$. Bipolar-Valued fuzzy sets have membership degrees that represent the degree of satisfaction to the property and its counter property. In a bipolar valued fuzzy set the membership degree 0 means that elements are irrelevant to the corresponding property, the membership degrees on $(0,1]$ indicate that elements some what satisfy the property, and the membership degrees on $[-1,0)$ indicate that elements somewhat satisfy the implicit counter property $[\sec (3)]$. In the definition of bipolar-valued fuzzy sets, there are two kinds of representations so called canonical representation and reduced representation. In this paper, we use the canonical representation of bipolar valued Q- fuzzy sets. In this paper, The theory of fuzzy sets which was introduced by Zadeh [6] is applied to many mathematical branches. Abou-zoid [1] , introduced the notion of a fuzzy sub near-ring and studied fuzzy ideals of near-ring. This concept discussed by many researchers among cho, Davvaz, Dudek, Jun, Kim [2],[3],[4]. In [5], considered the intuitionistic fuzzification of a right (resp left ) R- subgroup in a near-ring. Also cho.at.al in [4] the notion of normal intuitionistic fuzzy R- subgroup in a nearring is introduced and related properties are investigated. The notion of intuitionistic Q- fuzzy semi primality in a semi group is given by Kim [3]. In this paper, We introduce the notion of Q- fuzzification of bipolar left R- subgroups in a near ring and investigate some related properties. Characterization of bipolar Q- fuzzy left R- subgroups are given.

\section{PRELIMINARIES}

2.1 Definition : A non empty set with two binary operations '+' and '.' is called a near-ring if it satisfies the following axioms

(i) $\quad(\mathrm{R},+)$ is a group. (ii) $\quad(\mathrm{R},$.$) is a semi group.$

(iii) $\quad \mathrm{x} \cdot(\mathrm{y}+\mathrm{z})=\mathrm{x} \cdot \mathrm{y}+\mathrm{x} \cdot \mathrm{z}$

for all $\mathrm{x}, \mathrm{y}, \mathrm{z} \in \mathrm{R}$. Precisely speaking it is a left near-ring. Because it satisfies the left distributive law.

As $R$ - subgroup of a near- ring ' $R$ ' is a subset ' $H$ ' of ' $R$ ' such that

(i) $\quad(\mathrm{H},+)$ is a subgroup of $(\mathrm{R},+)$.

(ii) $\mathrm{RH} \subset \mathrm{H}$

(iii) $\quad H R \subset H$. If ' $H$ ' satisfies (i) and (ii) then it is called left R- subgroup of ' $R$ ' and if ' $H$ ' satisfies (i) and (iii) then it is called a right $\mathrm{R}$ subgroup of ' $R$ '. A map $f: R \rightarrow S$ is called homomorphism if $f(x+y)=f(x)+f(y)$ for all $\mathrm{x}, \mathrm{y}$ in $\mathrm{R}$.

Definition 2.1: Let ' $S$ ' be a set. A Q- fuzzy set in $S$ is a function $\mu: \mathrm{S} \times \mathrm{Q} \rightarrow[0,1]$

Definition 2.2: Let ' $G$ ' be a non-empty set. A bipolarValued Q-Fuzzy set A in $\mathrm{G}$ is an object having the form. $\mathrm{A}=\left\{\left((\mathrm{x}, \mathrm{q}) \mu_{\mathrm{A}}^{+}(\mathrm{x}, \mathrm{q}), \mu_{\mathrm{A}}^{-}(\mathrm{x}, \mathrm{q})\right) / \mathrm{x} \varepsilon \mathrm{G}, \mathrm{q} \varepsilon \mathrm{Q}\right\}$ where $\mu_{\mathrm{A}}{ }^{+}: \mathrm{G} \times \mathrm{Q} \rightarrow[0,1]$ and $\mu_{\mathrm{A}}{ }^{+} \mathrm{G} \times \mathrm{Q} \rightarrow[-1,0]$ are mapping. The positive membership degree $\mu_{\mathrm{A}}^{+}(\mathrm{x}, \mathrm{q})$ denotes the satisfaction degree of an element $\mathrm{x}$ to the property corresponding to ' $\mathrm{A}$ ' and the negative membership degree $\mu_{\mathrm{A}}^{+}(\mathrm{x}, \mathrm{q})$ denotes the satisfaction degree of $\mathrm{x}$ to some implicit counter property of $\mathrm{A}$.

Definition 2.3: Let ' $R$ ' be a near ring. A fuzzy set ' $\mu$ ' in $R$ is called fuzzy subnear ring in ' $R$ ' if (i) $\mu(x-y) \geq \min \{$ $\mu(\mathrm{x}), \mu(\mathrm{y})\} \quad$ (ii) $\mu(\mathrm{xy}) \geq \min \{\mu(\mathrm{x}), \mu(\mathrm{y})\}$ for all $\mathrm{x}, \mathrm{y}$ in $\mathrm{R}$.

Definition2.4 : A 'Q'-fuzzy set ' $\mu$ ' is called a Bi polar Qfuzzy left R- subgroup of R over $Q$ if ' $\mu$ ' satisfies
(i) $\quad \mu^{+}(x-y, q) \geq \min \left\{\mu^{+}(x, q), \mu^{+}(y, q)\right\}$
(ii) $\quad \mu^{-}(\mathrm{x}-\mathrm{y}, \mathrm{q}) \leq \max \left\{\mu^{-}(\mathrm{x}, \mathrm{q}), \mu^{-}(\mathrm{y}, \mathrm{q})\right\}$
(iii) $\quad \mu^{+}(\mathrm{rx}, \mathrm{q}) \geq \mu^{+}\left(\mathrm{x}\right.$ q). (iv) $\mu^{-}(\mathrm{rx}, \mathrm{q})$

$$
=\mu^{-}(\mathrm{x} q) \text {. }
$$

Definition2,5: For a bipolar Q- fuzzy set 'A' and $(\beta, \alpha) \varepsilon$ $[-1.0] \times[0,1]$, we define

$$
\begin{aligned}
& \mathrm{A}_{\mathrm{t}}^{+}=\left\{\mathrm{x} \varepsilon \mathrm{X} / \mu_{\mathrm{A}}^{+}(\mathrm{x}, \mathrm{q}) \leq \alpha\right\} \\
& \mathrm{A}_{\mathrm{S}}{ }^{-}=\left\{\mathrm{x} \varepsilon \mathrm{X} / \mu_{\mathrm{A}}^{-}(\mathrm{x}, \mathrm{q}) \geq \alpha\right\}
\end{aligned}
$$

which are called the positive t-cut and negative s-cut of $\mathrm{A}$ respectively.

Definition 2.6: Let $\lambda$ and $\mu$ be two Q-fuzzy subsets in X. The Cartesian Product of $\lambda^{+} \times \mu^{+}: X \times X \rightarrow[0,1]$ is defined by 
$\lambda^{+} \times \mu^{+}(\mathrm{x}, \mathrm{y})_{\mathrm{q}}=\mathrm{T}\left\{\lambda^{+}(\mathrm{x}, \mathrm{q}), \mu^{+}(\mathrm{y}, \mathrm{q})\right\}$ and $\lambda^{+} \times \mu^{+}: X \times X \rightarrow[0,1]$ is defined by $\lambda^{+} \times \mu^{+}(\mathrm{x}, \mathrm{y})_{\mathrm{q}}=\mathrm{S}\left\{\lambda^{-}(\mathrm{x}, \mathrm{q}), \mu^{-}(\mathrm{y}, \mathrm{q})\right\}$ for all $\mathrm{x}, \mathrm{y} \& \mathrm{X}$ and $\mathrm{q} \varepsilon \mathrm{Q}$.

Definition 2.7: Let $\mathrm{f}: \mathrm{X} \rightarrow \mathrm{Y}$ be a mapping of groups and ' $\mu$ ' be a bipolar Q- fuzzy set of $Y$. The map $\mu^{\mathrm{f}}$ is the pre image of $\mu_{1}$ and $\mu_{2}$ under f. If $\mu_{1}{ }^{\mathrm{f}+}(\mathrm{x}, \mathrm{q})=\mu^{\mathrm{f+}}(\mathrm{x}, \mathrm{q}), \mu_{2}{ }^{\mathrm{f}-\mathrm{x}}(\mathrm{x}, \mathrm{q})=$ $\mu^{\mathrm{f}--}(\mathrm{x}, \mathrm{q})$.

\section{PROPERTIES OF Q- FUZZY LEFT R- SUBGROUPS}

Proposition 3.1: Every imaginable Bi polar Q- fuzzy left R- subgroup $\mu^{+}$of a near ring ' $\mathrm{S}$ ' is a Q-fuzzy left Rsubgroup of $\mathrm{S}$.

Proof: $\quad$ Assume $\mu^{+}$is imaginable bipolar

Q- fuzzy left R- subgroup of ' $S$ ', then we have

(i) $\mu^{+}(\mathrm{x}-\mathrm{y}, \mathrm{q}) \geq \mathrm{T}\left\{\mu^{+}(\mathrm{x}, \mathrm{q}), \mu^{+}(\mathrm{y}, \mathrm{q})\right\}$ and

$\mu^{+}(r x, q) \geq \mu^{+}(x, q)$ for all $x, y$ in $S$.

Since $\mu^{+}$is imaginable, we have

$\min \left\{\mu^{+}(\mathrm{x}, \mathrm{q}), \mu^{+}(\mathrm{y}, \mathrm{q})\right\}=\mathrm{T}\left\{\min \left\{\mu^{+}(\mathrm{x}, \mathrm{q}), \mu^{+}(\mathrm{y}, \mathrm{q}), \min \{\right.\right.$ $\left.\left.\mu^{+}(\mathrm{x}, \mathrm{q}), \mu^{+}(\mathrm{y}, \mathrm{q})\right\}\right\}$

$$
\begin{aligned}
\geq \mathrm{T}\left(\mu^{+}(\mathrm{x}, \mathrm{q}), \mu^{+}(\mathrm{y}, \mathrm{q})\right) \\
\geq \min \left\{\mu^{+}(\mathrm{x}, \mathrm{q}), \mu^{+}(\mathrm{y}, \mathrm{q})\right\}
\end{aligned}
$$

And so (ii) $\mathrm{T}\left(\mu^{+}(\mathrm{x}, \mathrm{q}), \mu^{+}(\mathrm{y}, \mathrm{q})\right)=$

$$
\begin{gathered}
\min \left\{\mu^{+}(\mathrm{x}, \mathrm{q}), \mu^{+}(\mathrm{y}, \mathrm{q})\right\} . \text { It follows that } \\
\mu^{+}(\mathrm{x}-\mathrm{y}, \mathrm{q}) \geq \mathrm{T}\left(\mu^{+}(\mathrm{x}, \mathrm{q}), \mu^{+}(\mathrm{y}, \mathrm{q})\right) \\
=\min \left\{\mu^{+}(\mathrm{x}, \mathrm{q}), \mu^{+}(\mathrm{y}, \mathrm{q})\right\} \\
\text { for all } \mathrm{x}, \mathrm{y} \varepsilon \mathrm{S} \text {. Hence } \mu^{+} \text {is a }
\end{gathered}
$$

Bipolar Q-fuzzy left R- subgroup of S

$$
\text { Also(iii) } \begin{aligned}
\mu^{--} & (\mathrm{x}-\mathrm{y}, \mathrm{q}) \\
& \leq \mathrm{S}\left\{\mu^{-}(\mathrm{x}, \mathrm{q}), \mu^{-}(\mathrm{y}, \mathrm{q})\right\} \text { and }
\end{aligned}
$$

$\mu^{-}(\mathrm{rx}, \mathrm{q}) \leq \mu^{-}(\mathrm{x}, \mathrm{q})$ for all $\mathrm{x}, \mathrm{y}$ in $\mathrm{S}$.

Since $\mu^{--}$is imaginable, we have

$\max \left\{\mu^{--}(\mathrm{x}, \mathrm{q}), \mu^{--}(\mathrm{y}, \mathrm{q})\right\}$

$=\mathrm{S}\left\{\max \left\{\mu^{--}(\mathrm{x}, \mathrm{q}), \mu^{--}(\mathrm{y}, \mathrm{q}), \max \left\{\mu^{--}(\mathrm{x}, \mathrm{q})\right.\right.\right.$,

$$
\begin{aligned}
& \leq \mathrm{S}\left(\mu^{--}(\mathrm{x}, \mathrm{q}), \mu^{--}(\mathrm{y}, \mathrm{q})\right) \\
& \leq \max \left\{\mu^{--}(\mathrm{x}, \mathrm{q}), \mu^{--}(\mathrm{y}, \mathrm{q})\right\}
\end{aligned}
$$$$
\left.\mu^{--}(\mathrm{y}, \mathrm{q})\right\}
$$

And so

(iv) $\quad \mathrm{S}\left(\mu^{--}(\mathrm{x}, \mathrm{q}), \mu^{--}(\mathrm{y}, \mathrm{q})\right)=\max \left\{\mu^{--}(\mathrm{x}, \mathrm{q})\right.$,

$$
\left.\mu^{--}(\mathrm{y}, \mathrm{q})\right\} \text {. It follows that }
$$

$$
\mu^{--}(\mathrm{x}-\mathrm{y}, \mathrm{q}) \leq \mathrm{S}\left(\mu^{--}(\mathrm{x}, \mathrm{q}), \mu^{--}(\mathrm{y}, \mathrm{q})\right)
$$

$$
=\max \left\{\mu^{--}(\mathrm{x}, \mathrm{q}), \mu^{--}(\mathrm{y}, \mathrm{q})\right\}
$$

for all $x, y \in S$. Hence $\mu^{--}$is a Bipolar Q-fuzzy left Rsubgroup of $\mathrm{S}$.

Proposition 3.2: If $\mu^{+}$is a Bipolar Q- fuzzy left Rsubgroups of a near ring ' $S$ ' and ' $\theta$ is an endomorphism of $S$, then $\mu[\Theta]$ is Bipolar

Q- fuzzy left R- subgroup of 'S'.

Proof: For any $\mathrm{x}, \mathrm{y} \varepsilon \mathrm{S}$, we have

$$
\text { (i) } \begin{aligned}
& \mu^{+}[\theta](\mathrm{x}-\mathrm{y}, \mathrm{q})=\mu^{+}(\theta(\mathrm{x}-\mathrm{y}, \mathrm{q})) \\
&= \mu^{+}(\theta(\mathrm{x}, \mathrm{q}), \theta(\mathrm{y}, \mathrm{q})) \\
& \geq \mathrm{T}\left\{\mu^{+}(\theta(\mathrm{x}, \mathrm{q})), \mu^{+}(\theta(\mathrm{y}, \mathrm{q}))\right\} \\
&=\mathrm{T}\left\{\mu^{+}[\theta](\mathrm{x}, \mathrm{q}), \mu^{+}[\theta](\mathrm{y}, \mathrm{q})\right\}
\end{aligned}
$$

$$
\text { (ii) } \begin{aligned}
\mu^{+}[\theta](\mathrm{rx}, \mathrm{q}) & =\mu^{+}(\theta(\mathrm{rx}, \mathrm{q}) \\
& \geq \mu^{+}(\theta(\mathrm{x}, \mathrm{q})) \\
& \geq \mu^{+}[\theta](\mathrm{x}, \mathrm{q}) .
\end{aligned}
$$

$$
\text { (iii) } \begin{aligned}
\mu^{--}[\theta] & (\mathrm{x}-\mathrm{y}, \mathrm{q})=\mu^{--}(\theta(\mathrm{x}-\mathrm{y}, \mathrm{q})) \\
& =\mu^{--}(\theta(\mathrm{x}, \mathrm{q}), \theta(\mathrm{y}, \mathrm{q})) \\
& \leq \mathrm{S}\left\{\mu^{--}(\theta(\mathrm{x}, \mathrm{q})), \mu^{--}(\theta(\mathrm{y}, \mathrm{q}))\right\}
\end{aligned}
$$

$$
=S\left\{\mu^{--}[\theta](\mathrm{x}, \mathrm{q}), \mu^{--}[\theta](\mathrm{y}, \mathrm{q})\right\}
$$

$$
\text { (iv) } \begin{aligned}
\mu^{--}[\theta](\mathrm{rx}, \mathrm{q}) & =\mu^{--}(\theta(\mathrm{rx}, \mathrm{q}) \\
& \leq \mu^{--}(\theta(\mathrm{x}, \mathrm{q})) \\
& \leq \mu^{--}[\theta](\mathrm{x}, \mathrm{q}) .
\end{aligned}
$$

Hence $\mu^{--}[\theta]$ is bipoloar Q- fuzzy left $\mathrm{R}$ - subgroup of $\mathrm{R}$.

Proposition 3.3: An onto homomorphism's of a bipolar Q- fuzzy left R- subgroup of near ring 'S' is bipolar Q- fuzzy left R-subgroup.

Proof: Let $\mathrm{f}: \mathrm{S} \rightarrow \mathrm{S}^{1}$ be an onto homomorphism of near rings and let ' $\xi$ ' be a Q- fuzzy bipolar left R- subgroup of $S^{1}$ and $\mu^{+}$be the pre image of ' $\xi$ ' under ' $f$ ', then we have

$$
\begin{aligned}
\text { (i) } \mu^{+}(\mathrm{x}-\mathrm{y}, \mathrm{q}) & =\xi(\mathrm{f}(\mathrm{x}-\mathrm{y}, \mathrm{q})) \\
& =\xi(\mathrm{f}(\mathrm{x}, \mathrm{q}), \mathrm{f}(\mathrm{y}, \mathrm{q})) \\
& \geq \mathrm{T}(\xi(\mathrm{f}(\mathrm{x}, \mathrm{q})), \xi(\mathrm{f}(\mathrm{y}, \mathrm{q}))) \\
& \geq \mathrm{T}\left(\mu^{+}(\mathrm{x}, \mathrm{q}), \mu^{+}(\mathrm{y}, \mathrm{q})\right) \\
& =\xi(\mathrm{f}(\mathrm{rx}, \mathrm{q})) \\
& \geq \xi(\mathrm{f}(\mathrm{x}, \mathrm{q})) \\
& \geq \mu^{+}(\mathrm{x}, \mathrm{q}) . \\
& =\xi(\mathrm{f}(\mathrm{x}-\mathrm{y}, \mathrm{q})) \\
& =\xi(\mathrm{f}(\mathrm{x}, \mathrm{q}), \mathrm{f}(\mathrm{y}, \mathrm{q})) \\
& \leq \mathrm{S}(\xi(\mathrm{f}(\mathrm{x}, \mathrm{q})), \xi(\mathrm{f}(\mathrm{y}, \mathrm{q}))) \\
& \leq \mathrm{S}\left(\mu^{--}(\mathrm{x}, \mathrm{q}), \mu^{--}(\mathrm{y}, \mathrm{q})\right) \\
\text { (iv) } \mu^{--}(\mathrm{rx}, \mathrm{q}) & =\xi(\mathrm{f}(\mathrm{rx}, \mathrm{q})) \\
& \leq \xi(\mathrm{f}(\mathrm{x}, \mathrm{q})) \\
& \leq \mu^{--}(\mathrm{x}, \mathrm{q}) .
\end{aligned}
$$

Proposition 3.4: An onto homomorphic image of a bipolar Q- fuzzy left R- subgroup with the sup property is a bipolar Q- fuzzy left R- subgroup.

Proof: Let $\mathrm{f}: \mathrm{S} \rightarrow \mathrm{S}^{1}$ be an onto homomorphism of near rings and let $\mu^{+}$be a sup property of fuzzy left R- subgroups of ' $S$ '. Let $\mathrm{x}^{1}, \mathrm{y}^{1} \varepsilon \mathrm{S}^{1}$, and $\mathrm{x}_{0} \varepsilon \mathrm{f}^{-1}\left(\mathrm{x}^{1}\right), \mathrm{y}_{0} \varepsilon \mathrm{f}^{1}\left(\mathrm{y}^{1}\right)$ be such that

$$
\left.\mu^{\mathrm{f}+}\left(\mathrm{x}_{0}, \mathrm{q}\right)=\sup _{(\mathrm{h}, \mathrm{q}) \varepsilon \mathrm{f}^{-1}\left(\mathrm{x}^{1}\right)} \mu^{+}(\mathrm{h}, \mathrm{q}), \operatorname{Sup}^{+}\left(\mathrm{y}_{0}, \mathrm{q}\right) \mathrm{q}\right) \varepsilon \mathrm{f}^{-1}\left(\mathrm{y}^{1}\right)
$$

respectively, then we can deduce that

$$
\begin{aligned}
& \text { (i) } \mu^{\mathrm{f}+}\left(\mathrm{x}^{1}-\mathrm{y}^{1}, \mathrm{q}\right) \\
& =\sup \mu^{+}(\mathrm{z}, \mathrm{q}),(\mathrm{z}, \mathrm{q}) \varepsilon \mathrm{f}^{-1}\left(\mathrm{x}^{1}-\mathrm{y}^{1}, \mathrm{q}\right) \\
& \geq \min \left\{\mu^{+}\left(\mathrm{x}_{0}, \mathrm{q}\right), \mu^{+}\left(\mathrm{y}_{0}, \mathrm{q}\right)\right. \\
& =\min \left\{\sup \mu^{+}(h, q), \sup \mu^{+}(h, q)\right\} \\
& =\min \left\{\mu^{+} \mathrm{f}\left(\mathrm{x}^{1}, \mathrm{q}\right), \mu^{+} \mathrm{f}\left(\mathrm{y}^{1}, \mathrm{q}\right)\right\} \\
& (\mathrm{h}, \mathrm{q}) \varepsilon \mathrm{f}^{-1}\left(\mathrm{x}^{1}, \mathrm{q}\right) \quad(\mathrm{h}, \mathrm{q}) \varepsilon \mathrm{f}^{-1}\left(\mathrm{y}^{1}, \mathrm{q}\right)
\end{aligned}
$$

(ii) $\mu^{\mathrm{f}+}(\mathrm{rx}, \mathrm{q})=\sup \mu^{+}(\mathrm{z}, \mathrm{q})$

$$
(\mathrm{z}, \mathrm{q}) \varepsilon \mathrm{f}^{-1}\left(\mathrm{r}^{1} \mathrm{x}^{1}, \mathrm{q}\right)
$$$$
\geq \mu^{+}\left(\mathrm{y}_{0}, \mathrm{q}\right)
$$$$
=\sup \mu^{+}(\mathrm{h}, \mathrm{q})
$$$$
(\mathrm{h}, \mathrm{q}) \varepsilon \mathrm{f}^{-1}\left(\mathrm{y}^{1}, \mathrm{q}\right)
$$$$
=\mu^{\mathrm{f}+}\left(\mathrm{y}^{1}, \mathrm{q}\right)
$$$$
\text { (iv) } \quad \mu^{\mathrm{f}--}\left(\mathrm{x}^{1}-\mathrm{y}^{1}, \mathrm{q}\right)
$$

$$
\begin{aligned}
& =\inf \mu^{--}(\mathrm{z}, \mathrm{q}),(\mathrm{z}, \mathrm{q}) \varepsilon \mathrm{f}^{-1}\left(\mathrm{x}^{1}-\mathrm{y}^{1}, \mathrm{q}\right) \\
& \leq \max \left\{\mu^{--}\left(\mathrm{x}_{0}, \mathrm{q}\right), \mu^{--}\left(\mathrm{y}_{0}, \mathrm{q}\right)\right. \\
& =\max \left\{\inf \mu^{--}(\mathrm{h}, \mathrm{q}), \inf \mu^{--}(\mathrm{h}, \mathrm{q})\right\} \\
& \quad(\mathrm{h}, \mathrm{q}) \varepsilon \mathrm{f}^{1}\left(\mathrm{x}^{1}, \mathrm{q}\right) \quad(\mathrm{h}, \mathrm{q}) \varepsilon \mathrm{f}^{1}\left(\mathrm{y}^{1}, \mathrm{q}\right) \\
& =\max \left\{\mu^{--} \mathrm{f}\left(\mathrm{x}^{1}, \mathrm{q}\right), \mu^{--} \mathrm{f}\left(\mathrm{y}^{1}, \mathrm{q}\right)\right\}
\end{aligned}
$$

$$
\text { (iv) } \begin{aligned}
\mu^{\mathrm{f}---}(\mathrm{rx}, \mathrm{q}) & =\quad \inf _{(\mathrm{z}, \mathrm{q}) \varepsilon \mathrm{f}^{-1}\left(\mathrm{r}^{1} \mathrm{x}^{1}, \mathrm{q}\right)} \\
& \leq \quad \mu^{--}\left(\mathrm{y}_{0}, \mathrm{q}\right) \\
& =\inf \mu^{--}(\mathrm{h}, \mathrm{q})
\end{aligned}
$$




$$
=\begin{aligned}
& (\mathrm{h}, \mathrm{q}) \varepsilon \mathrm{f}^{1}\left(\mathrm{y}^{1}, \mathrm{q}\right) \\
& \mu^{\mathrm{f}--}\left(\mathrm{y}^{1}, \mathrm{q}\right) .
\end{aligned}
$$

Hence ${ }^{\mathrm{ft}}{ }^{\mathrm{f+}}$ is a bipolar Q-fuzzy left R- subgroup of $S^{1}$.

Proposition 3.5: Let ' $\mathrm{T}$ ' be a continuous tnorm and let ' $\mathrm{f}$ ' be a homomorphism on a near ring ' $\mathrm{S}$ '. If ' $\mu$ ', is bipolar Q- fuzzy left $R$ - subgroup of $S$, then $\mu^{+f}$ is a bipolar Q- fuzzy left R- subgroup of $\mathrm{f}(\mathrm{S})$.

Proof: Let $A_{1}=f^{-1}\left(y_{1}, q\right), A_{2}=f^{-1}\left(y_{2}, q\right)$ and

$A_{12}=f^{-1}\left(y_{1}-y_{2}, q\right)$ where $y_{1}, y_{2} \& f(S), q \varepsilon Q$. consider the set

$\mathrm{A}_{1}-\mathrm{A}_{2}=\left\{\mathrm{x} \varepsilon \mathrm{S} /(\mathrm{x}, \mathrm{q})=\left(\mathrm{a}_{1}, \mathrm{q}\right)-\left(\mathrm{a}_{2}, \mathrm{q}\right)\right\}$ for some $\left(\mathrm{a}_{1}, \mathrm{q}\right) \varepsilon \mathrm{A}_{1}$ and $\left(\mathrm{a}_{2}, \mathrm{q}\right) \varepsilon \mathrm{A}_{2}$.

If $(x, q) \& A_{1}-A_{2}$, then $(x, q)=\left(x_{1}, q\right)-\left(x_{2}, q\right)$ for some

$\left(\mathrm{x}_{1}, \mathrm{q}\right) \& \mathrm{~A}_{1}$ and $\left(\mathrm{x}_{2}, \mathrm{q}\right) \& \mathrm{~A}_{2} \quad$ so that we have

$f(x, q)=f\left(x_{1}, q\right)-f\left(x_{2}, q\right)$

$$
=\mathrm{y}_{1}-\mathrm{y}_{2}
$$

(ie) $(\mathrm{x}, \mathrm{q}) \varepsilon \mathrm{f}^{-1}\left(\left(\mathrm{y}_{1}, \mathrm{q}\right)-\left(\mathrm{y}_{2}, \mathrm{q}\right)\right)=\mathrm{f}^{-1}\left(\mathrm{y}_{1}-\mathrm{y}_{2}, \mathrm{q}\right)$

$\mathrm{A}_{12}$.

$$
=A_{12} \text {. Thus } \quad A_{1}-A_{2} c
$$

It follows that

$$
\text { (i) } \begin{aligned}
& \mu^{\mathrm{f}+}\left(\mathrm{y}_{1}-\mathrm{y}_{2}, \mathrm{q}\right) \\
&\left.=\sup \left\{\mu^{+}(\mathrm{x}, \mathrm{q}) /(\mathrm{x}, \mathrm{q}) \varepsilon \mathrm{f}^{-1}\left({ }_{(\mathrm{y}}, \mathrm{q}\right)-\left(\mathrm{y}_{2}, \mathrm{q}\right)\right)\right\} \\
& \sup \left\{\mu^{+}(\mathrm{x}, \mathrm{q}) /(\mathrm{x}, \mathrm{q}) \varepsilon \mathrm{A}_{12}\right\} \\
& \geq \sup \left\{\mu^{+}(\mathrm{x}, \mathrm{q}) /(\mathrm{x}, \mathrm{q}) \varepsilon \mathrm{A}_{1}-\mathrm{A}_{2}\right\} \\
& \geq \sup \left\{\mu^{+}\left(\left(\mathrm{x}_{1}, \mathrm{q}\right)-\left(\mathrm{x}_{2}, \mathrm{q}\right)\right) /\right. \\
&\left.\left(\mathrm{x}_{1}, \mathrm{q}\right) \varepsilon \mathrm{A}_{1} \text { and }\left(\mathrm{x}_{2}, \mathrm{q}\right) \varepsilon \mathrm{A}_{2}\right\} \\
& \geq \sup \left\{\mathrm{T}\left(\mu^{+}\left(\mathrm{x}_{1}, \mathrm{q}\right), \mu^{+}\left(\mathrm{x}_{2}, \mathrm{q}\right)\right) /\right. \\
&\left.\quad\left(\mathrm{x}_{1}, \mathrm{q}\right) \& \mathrm{~A}_{1} \text { and }\left(\mathrm{x}_{2}, \mathrm{q}\right) \varepsilon \mathrm{A}_{2}\right\}
\end{aligned}
$$

Since ' $T$ ' is continuous. For every $\varepsilon>0$, we see that if

$\left.\sup \left\{\mu^{+}\left(\mathrm{x}_{1}, \mathrm{q}\right) /\left(\mathrm{x}_{1}, \mathrm{q}\right) \varepsilon \mathrm{A}_{1}\right\}-\left(\mathrm{x}_{1}^{*}, \mathrm{q}\right)\right\} \leq \delta$ and

$\left.\sup \left\{\mu^{+}\left(\mathrm{x}_{2}, \mathrm{q}\right) /\left(\mathrm{x}_{2}, \mathrm{q}\right) \varepsilon \mathrm{A}_{2}\right\}-\left(\mathrm{x}_{2}{ }^{*}, \mathrm{q}\right)\right\} \leq \delta$

$\mathrm{S}\left\{\sup \left\{\mu^{+}\left(\mathrm{x}_{1}, \mathrm{q}\right) /\left(\mathrm{x}_{1}, \mathrm{q}\right) \varepsilon \mathrm{A}_{1}\right\}, \sup \left\{\mu^{+}\left(\mathrm{x}_{2,}, \mathrm{q}\right) /\right.\right.$ $\left.\left(\mathrm{x}_{2}, \mathrm{q}\right) \varepsilon \mathrm{A}_{2}\right\}-\mathrm{T}\left(\left(\mathrm{x}_{1}{ }^{*}, \mathrm{q}\right),\left(\mathrm{x}_{2}{ }^{*}, \mathrm{q}\right) \leq \varepsilon\right.$

Choose $\left(\mathrm{a}_{1}, \mathrm{q}\right) \& \mathrm{~A}_{1}$ and $\left(\mathrm{a}_{2}, \mathrm{q}\right) \& \mathrm{~A}_{2}$ such that $\sup \left\{\mu^{+}\left(\mathrm{x}_{1}, \mathrm{q}\right) /\left(\mathrm{x}_{1} . \mathrm{q}\right) \& \mathrm{~A}_{1}\right\}-\mu^{+}\left(\mathrm{a}_{1}, \mathrm{q}\right) \leq \delta \quad$ and $\sup \left\{\mu^{+}\left(\mathrm{x}_{2}, \mathrm{q}\right) /\left(\mathrm{x}_{2}, \mathrm{q}\right) \varepsilon \mathrm{A}_{2}\right\}-\mu\left(\mathrm{a}_{2}, \mathrm{q}\right) \leq \delta$. Then we have $\mathrm{T}\left\{\sup \left\{\mu^{+}\left(\mathrm{x}_{1}, \mathrm{q}\right) /\left(\mathrm{x}_{1}, \mathrm{q}\right) \varepsilon \mathrm{A}_{1}\right\}, \sup \left\{\mu^{+}\left(\mathrm{x}_{2}, \mathrm{q}\right) /\right.\right.$ $\left.\left(\mathrm{x}_{2}, \mathrm{q}\right) \varepsilon \mathrm{A}_{2}\right\}-\mathrm{T}\left(\mu\left(\mathrm{a}_{1}, \mathrm{q}\right), \mu\left(\mathrm{a}_{2}, \mathrm{q}\right) \leq \varepsilon\right.$ consequently, we have

$$
\begin{aligned}
& \left.\mu^{\mathrm{f}+} \mathrm{y}_{1}-\mathrm{y}_{2}, \mathrm{q}\right) \geq \sup \left\{\mathrm{T}\left(\mu^{+}\left(\mathrm{x}_{1}, \mathrm{q}\right), \mu^{+}\left(\mathrm{x}_{2}, \mathrm{q}\right)\right)\right. \\
& \left./\left(\mathrm{x}_{1}, \mathrm{q}\right) \& \mathrm{~A}_{1},\left(\mathrm{x}_{2}, \mathrm{q}\right) \& \mathrm{~A}_{2}\right\} \\
& \geq \mathrm{T}\left(\inf \left\{\mu^{+}\left(\mathrm{x}_{1}, \mathrm{q}\right) /\left(\mathrm{x}_{1}, \mathrm{q}\right) \varepsilon \mathrm{A}_{1}\right\}, \inf \left\{\mu^{+}\right.\right. \\
& \left.\left(\mathrm{x}_{2}, \mathrm{q}\right) /\left(\mathrm{x}_{2}, \mathrm{q}\right) \varepsilon \mathrm{A}_{2}\right\} \\
& \text { (ii) } \mu^{\mathrm{f}--}\left(\mathrm{y}_{1}-\mathrm{y}_{2}, \mathrm{q}\right) \\
& \geq \mathrm{T}\left(\mu^{\mathrm{f+}}\left(\mathrm{y}_{1}, \mathrm{q}\right), \mu^{\mathrm{ff}}\left(\mathrm{y}_{2}, \mathrm{q}\right)\right\} \\
& =\inf \left\{\mu^{--}(\mathrm{x}, \mathrm{q}) /(\mathrm{x}, \mathrm{q}) \varepsilon \mathrm{f}^{1}\left(\left(\mathrm{y}_{1}, \mathrm{q}\right)-\right.\right. \\
& =\inf \left\{\mu^{--}(\mathrm{x}, \mathrm{q}) /(\mathrm{x}, \mathrm{q}) \varepsilon \mathrm{A}_{12}\right\} \\
& \leq \inf \left\{\mu^{--}(\mathrm{x}, \mathrm{q}) /(\mathrm{x}, \mathrm{q}) \varepsilon \mathrm{A}_{1}-\mathrm{A}_{2}\right\} \\
& \leq \inf \left\{\mu^{--}\left(\left(x_{1}, q\right)-\left(x_{2}, q\right)\right) /\right. \\
& \left.\left(\mathrm{x}_{1}, \mathrm{q}\right) \& \mathrm{~A}_{1} \text { and }\left(\mathrm{x}_{2}, \mathrm{q}\right) \& \mathrm{~A}_{2}\right\} \\
& \leq \inf \left\{\mathrm{S}\left(\mu^{-}\left(\mathrm{x}_{1}, \mathrm{q}\right), \mu^{-}\left(\mathrm{x}_{2}, \mathrm{q}\right)\right) /\right. \\
& \left.\left(\mathrm{x}_{1}, \mathrm{q}\right) \& \mathrm{~A}_{1} \text { and }\left(\mathrm{x}_{2}, \mathrm{q}\right) \& \mathrm{~A}_{2}\right\}
\end{aligned}
$$

Since ' $\mathrm{S}$ ' is continuous. For every $\varepsilon>0$,

$$
\text { we see that if }
$$$$
\left.\operatorname{Inf}\left\{\mu\left(\mathrm{x}_{1}, \mathrm{q}\right) /\left(\mathrm{x}_{1}, \mathrm{q}\right) \varepsilon \mathrm{A}_{1}\right\}-\quad\left(\mathrm{x}_{1}{ }^{*}, \mathrm{q}\right)\right\} \leq \delta
$$

$\left.\operatorname{Inf}\left\{\mu\left(x_{2}, q\right) /\left(x_{2}, q\right) \varepsilon A_{2}\right\}-\left(x_{2}{ }^{*}, q\right)\right\} \leq \delta$

$S\left\{\inf \left\{\mu^{-}\left(x_{1}, q\right) /\left(x_{1}, q\right) \varepsilon A_{1}\right\}, \inf \left\{\mu^{+}\left(x_{2}, q\right) /\right.\right.$

$$
\left.\left(\mathrm{x}_{2}, \mathrm{q}\right) \varepsilon \mathrm{A}_{2}\right\}-\mathrm{S}\left(\left(\mathrm{x}_{1}{ }^{*}, \mathrm{q}\right),\left(\mathrm{x}_{2}{ }^{*}, \mathrm{q}\right) \leq \varepsilon\right.
$$

Choose $\left(a_{1}, q\right) \& A_{1}$ and $\left(a_{2}, q\right) \& A_{2}$ such that

Inf $\left\{\mu^{-}\left(\mathrm{x}_{1}, \mathrm{q}\right) /\left(\mathrm{x}_{1} . \mathrm{q}\right) \varepsilon \mathrm{A}_{1}\right\}-\mu^{+}\left(\mathrm{a}_{1}, \mathrm{q}\right) \leq \delta$ and

$\operatorname{Inf}\left\{\mu^{-}\left(\mathrm{x}_{2}, \mathrm{q}\right) /\left(\mathrm{x}_{2}, \mathrm{q}\right) \varepsilon \mathrm{A}_{2}\right\}-\mu\left(\mathrm{a}_{2}, \mathrm{q}\right) \leq \delta$.
Then we have

$\mathrm{S}\left\{\inf \left\{\mu^{-}\left(\mathrm{x}_{1}, \mathrm{q}\right) /\left(\mathrm{x}_{1}, \mathrm{q}\right) \varepsilon \mathrm{A}_{1}\right\}, \inf \left\{\mu^{-}\left(\mathrm{x}_{2}, \mathrm{q}\right) /\left(\mathrm{x}_{2}, \mathrm{q}\right) \varepsilon \mathrm{A}_{2}\right\}-\right.$ $\mathrm{S}\left(\mu^{+}\left(\mathrm{a}_{1}, \mathrm{q}\right), \mu\left(\mathrm{a}_{2}, \mathrm{q}\right) \leq \varepsilon\right.$

consequently, we have

$$
\mu^{\mathrm{f}--}\left(\mathrm{y}_{1}-\mathrm{y}_{2}, \mathrm{q}\right)
$$

$$
\begin{gathered}
\leq \inf \left\{\mathrm{S}\left(\mu^{-}\left(\mathrm{x}_{1}, \mathrm{q}\right), \mu^{-}\left(\mathrm{x}_{2}, \mathrm{q}\right)\right) /\left(\mathrm{x}_{1}, \mathrm{q}\right) \varepsilon\right. \\
\left.\mathrm{A}_{1},\left(\mathrm{x}_{2}, \mathrm{q}\right) \varepsilon \mathrm{A}_{2}\right\} \\
\leq \mathrm{S}\left(\inf \left\{\mu^{--}\left(\mathrm{x}_{1}, \mathrm{q}\right) /\left(\mathrm{x}_{1}, \mathrm{q}\right) \varepsilon \mathrm{A}_{1}\right\},\right. \\
\quad \inf \left\{\mu^{--}\left(\mathrm{x}_{2}, \mathrm{q}\right) /\left(\mathrm{x}_{2}, \mathrm{q}\right) \varepsilon A_{2}\right\} \\
\leq \mathrm{S}\left(\mu^{+\mathrm{f}}\left(\mathrm{y}_{1}, \mathrm{q}\right), \mu^{-\mathrm{f}}\left(\mathrm{y}_{2}, \mathrm{q}\right)\right\} .
\end{gathered}
$$

Hence ' $\mu$ ' is a bipolar Q- fuzzy left R- subgroup of ' $\mathrm{f}(\mathrm{S})$ '.

\section{CONCLUSION}

Osman kazanci , Sultanyamark and Serifeyilmaz(5) introduced the intutionistic Q- fuzzy R-subgroups of near rings. In this paper we investigate the notion of bipolar Qfuzzy left R- subgroup of near ring with respecti to(T,S)and characterization of them.

\section{REFERENCES}

[1] S. Abou-Zoid, " On fuzzy sub near rings and ideals", Fuzzy sets. Syst. 44 (1991), 139-146.

[2] Y.U. Cho, Y.B.Jun, “ On intuitionistic fuzzy R- subgroup of near rings", J. Appl. Math. And computing , 18 (1-2) (2005), 665-677.

[3] K.H.Kim , Y.B. Jun, "On fuzzy R- subgroups of near rings, J. fuzzy math 8 (3) (2000) 549-558.

[4] K.H.Kim, Y.B.Jun, “ Normal fuzzy R- subgroups of near rings , J. fuzzy sets. Syst.121(2001) 341-345.

[5] Osman Kazanci, Sultan Yamark and Serife Yimaz “ On intuitionistic Q- fuzzy R- subgroups of near rings, International mathematical forum , 2, 2007 , 59 , (28992910)

[6] A.Solairaju and R.Nagarajan, "Q-fuzzy left R- subgroup of near rings with respect to T-norms" Antarctica Journal Of Mathematics, 5(2)(2008)59-63.

[7] A.Solairaju and R.Nagarajan, "Lattice valued Q- fuzzy Left R- submodules of near rings with respect to Tnorms" Advances in Fuzzy Mathematics, 4(2)(2009),137-145.

[8] A.Solairaju and R.Nagarajan,"A New Structure and Constructions of Q- fuzzy groups" Advances in Fuzzy in Mathematica,4(1)(2009), 23-29.

[9] R.Nagarajan and A.Solairaju,"Characterization of anti Qfuzzy R-submodules over Commutative rings", International Journal of Computer Applications,5(3)(2010), 3-7.

[10] L.A.Zadeh,Fuzzy set, inform.control 8 (1965) 338-353. 